\title{
Effects of repetitive transcranial magnetic stimulation on clinical, social, and cognitive performance in postpartum depression
}

This article was published in the following Dove Press journal:

Neuropsychiatric Disease and Treatment

24 October 2012

Number of times this article has been viewed

\author{
Martin Luiz Myczkowski' \\ Álvaro Machado Dias ${ }^{1,2}$ \\ Tatiana Luvisotto' \\ Debora Arnaut' \\ Bianca Boura Bellini' \\ Carlos Gustavo Mansur' \\ Joel Rennó \\ Gabriel Tortella' \\ Philip Leite Ribeiro' \\ Marco Antônio Marcolin' \\ 'Institute of Psychiatry, University of \\ São Paulo, Medical School São Paulo, \\ ${ }^{2}$ Clinical Neuroscience Laboratory of \\ the Federal University of São Paulo, \\ São Paulo, Brazil
}

Background: This randomized, placebo-controlled, double-blind pilot study evaluated the impact of repetitive transcranial magnetic stimulation (rTMS) on clinical, cognitive, and social performance in women suffering with postpartum depression.

Methods: Fourteen patients were randomized to receive 20 sessions of sham rTMS or active $5 \mathrm{~Hz}$ rTMS over the left dorsolateral prefrontal cortex. Psychiatric clinical scales and a neuropsychological battery were applied at baseline (pretreatment), week 4 (end of treatment), and week 6 (follow-up, posttreatment week 2).

Results: The active rTMS group showed significant improvement 2 weeks after the end of rTMS treatment (week 6) in Hamilton Depression Rating Scale $(P=0.020)$, Global Assessment Scale $(P=0.037)$, Clinical Global Impression $(P=0.047)$, and Social Adjustment Scale-Self Report-Work at Home $(P=0.020)$.

Conclusion: This study suggests that rTMS has the potential to improve the clinical condition in postpartum depression, while producing marginal gains in social and cognitive function.

Keywords: transcranial magnetic stimulation, postpartum depression, clinical performance, cognitive performance, social performance

\section{Introduction}

Postpartum depression (PPD) is a depressive condition that can start within the first 4 weeks after delivery and shows the same nosological dimensions as major depression, eg, low mood, increased vulnerability to stress, social impairment, and cognitive deficits of relative importance, in association with an increased rate of dysphoric symptoms. ${ }^{1}$

PPD often represents a type of major depressive episode. Currently, it is believed that major depression postpartum affects around $7 \%$ of all women, whereas the inclusion of minor depression raises this rate to $19 \% .^{2}$ However, these numbers may be underestimated; the prevalence of the disorder is up to $60 \%$ in certain developing countries, which seldom receive the same attention as developed countries based on international epidemiological studies. ${ }^{3}$

Leaving aside the risk factors shared with major depression (eg, carrying one or more copies of the $s$ allele of 5-HTTLPR), ${ }^{4}$ as well as previous depressive episodes, vulnerability to PPD has mostly been associated with unintended pregnancy, domestic violence, single status, and poor-quality relationships. ${ }^{5}$

This disorder not only represents an ominous condition for the mother (to a lesser extent it may also affect the father), ${ }^{6}$ but it can also have deleterious effects on the child. ${ }^{7}$ Studies have suggested that PPD can affect birth outcomes and neonatal health, 
lead to future decreases in the infant's self-esteem, impair the establishment of social boundaries, decrease academic achievement, and increase twofold the risk of depression during childhood and adolescence. ${ }^{8,9}$

There are several hypotheses about the pathway that leads to the transmission of such effects. One perspective that has received much attention is that it involves both innate vulnerability and inappropriate learning, generated by exposure to maladaptive cognition and depressive affect. This inappropriate cognition and affect is often tied to the inability to cope with the mental and practical pressures represented by readjustment of family organization, as revealed by a recent study which found that multiple births dramatically increase $(45 \%)$ the chances of developing PPD. ${ }^{10}$ This perspective underscores the importance of enhancing the social and cognitive properties of the minds of patients suffering from PPD, in order to promote quality of life for the family and mitigate the impact on the child. ${ }^{11}$

PPD continues to be underdiagnosed and undertreated, despite increased awareness. Several risk factors have been identified, including anxiety or depression during pregnancy, a history of depression, and increased life stressors. Treatment for depression has been found to be mandatory during pregnancy and while breastfeeding for the quality of life of the family. However, the risks and benefits of specific treatment strategies must be carefully evaluated and balanced, in light of the particular features of the period. ${ }^{12}$ This perspective speaks directly to the cost/benefits of psychiatric medications in the treatment of PPD. ${ }^{13}$

These cost/benefits must take into consideration the potential risks to the newborn, which remain under debate (“... although rates of adverse events are low, serum level of antidepressants in the infant can vary widely, and the long-term outcome of infants exposed during breastfeeding is unknown"). ${ }^{14}$ It is also worth considering that the time window of lactation is narrow, whereas antidepressants usually take around 3-8 weeks to produce any phenomenological effect; finally, records provided to the US Food and Drug Administration show that even the most modern antidepressants show unimpressive clinical efficacy in certain depressive conditions. ${ }^{15}$ This situation highlights the need for methods to replace medication, while considering the perspective that psychotherapy alone is less effective than when combined with pharmacological treatment. ${ }^{16}$

The traditional option is electroconvulsive therapy, which is not only viewed as being aggressive, mostly because it leads to transient (and maybe long-term) cognitive impairment, but can also turn out to be particularly counterproductive in this context. ${ }^{17}$ Thus, in this sense, a potentially interesting alternative is represented by repetitive transcranial magnetic stimulation (rTMS), which yields immediate effects of a similar intensity to that of electroconvulsive therapy, ${ }^{18}$ but is safe (low risk of seizures and cognitive impairment) and much less aggressive. ${ }^{19}$ The technique is based on induction of focal electrical fields in the brain through magnetic pulses that are generated by an insulated metal coil placed over the scalp. ${ }^{20,21}$ These electrical fields augment the efficacy of neurotransmission, increase metabolic rates in related neuronal tissues, and induce expression of genes that extend the duration of near-immediate effects. ${ }^{22}$

Over the last decade, an increasing number of studies have investigated the potential of rTMS as an aid in the treatment of depression; from 2001 to 2011, 12 meta-analyses of the antidepressant efficacy of rTMS were indexed in PubMed. The first one was published by Holtzheimer et al, ${ }^{23}$ while the most recent one was published by Schutter, who concluded that active rTMS has an effect size of $0.63 .{ }^{24}$

According to another recent uncontrolled study on the efficacy of rTMS in the treatment of PPD, nine antidepressant-free women with PPD were given 20 sessions of rTMS treatment over 4 weeks $(10 \mathrm{~Hz}, 120 \%$ motor threshold, left dorsolateral prefrontal cortex). Duration of effect was assessed at 30 days, and at 3 months and 6 months after treatment. Multiple characteristics were assessed at baseline and throughout treatment. Assessments included the Hamilton Rating Scale for Depression-24 items, Edinburgh Postnatal Depression Scale (EPDS), Inventory of Depressive Symptomatology-Self-Report, and Clinical Global Impression-Severity scores to compare performances at four time points (baseline, end of week 2, end of week 4, and 180-day follow-up). Significant effects were noticed after only 2 weeks of treatment, with eight out of nine patients remitting from depression after 4 weeks, and seven out of these eight patients remaining in remission after 180 days. Further randomized, sham-controlled studies need to be completed. ${ }^{25}$ Controlled studies with the same objective and scope have not been published so far, nor have studies addressing changes in cognitive and social performances.

The importance of appropriate cognitive and social strategies for coping with the challenge of parenting is evident, and it is interesting to note that a recent meta-analysis suggested that rTMS increases cognitive function in individuals suffering from major depression. ${ }^{26}$ This effect probably relates to the fact that rTMS selectively modulates gamma-band oscillations $(30-50 \mathrm{~Hz})$ in the prefrontal cortex (measured using electroencephalography) through stimulation of fast-spiking 
GABAergic neurons that synchronize the pyramidal cells of the neural networks needed for proper performance in different types of working memory tasks. ${ }^{27}$ In this sense, it has been argued that rTMS can produce cognitive enhancement in normal working memory tasks, in association with increases in neural efficiency. ${ }^{28}$

In relation to the potential positive consequences of rTMS on social performance and its association with clinical and cognitive performance, relevant data are notably scarce. As revealed by our thorough review of the literature indexed in PubMed and Institute of Scientific Information, there is not a single experimental study addressing these questions to date. This is somewhat surprising, considering that several etiological models of depression are based upon the assumption that the disorder impacts social abilities and cognitive functions ${ }^{29}$ and that, at a deep evolutionary level, it may have evolved as a cry for help, which may be particularly important in PPD. ${ }^{30}$ In this sense, we believe that an evaluation of social performance in treatment of PPD with rTMS is important and that these data should be considered in light of clinical and cognitive results.

Considering the potentially positive cost/benefits of rTMS in PPD, the current double-blind, randomized, controlled pilot study was primarily designed to evaluate the efficacy of active rTMS (in comparison with sham rTMS), with a focus on clinical conditions, cognitive abilities, and social performance.

\section{Materials and methods Sample}

The study sample consisted of 14 women of mean age 28.15 (range 18-36) years who had given birth 1-6 months (mean age of newborns 3.81 months) before the beginning of this clinical trial and who were diagnosed with major depression with a puerperal onset, according to the criteria of the DSM-IV-TR (Diagnostic and Statistical Manual of Mental Disorders, Fourth Edition, Text Revision) as well as through a structured clinical interview. ${ }^{31}$ None of the participants were receiving any pharmacological treatment other than clonazepam $1 \mathrm{mg}$ /day for insomnia and/or physical symptoms. Specifically, there were three patients allocated to the active group, whose insomnia and physical symptoms were more intense than average, eg, heart palpitations and headaches, therefore requiring benzodiazepines.

\section{Measures and procedures}

The eligibility criteria included baseline scores of at least 14 points on the 17 item-Hamilton Depression Rating
Scale (HDRS/17) $)^{32}$ and at least 13 points on the EPDS. ${ }^{33}$ Exclusion criteria included ferromagnetic metallic implants, pacemakers, previous neurosurgery, history of seizures, major head trauma, alcoholism, drug addiction, any psychiatric or neurological disorder other than depression and anxiety, psychotic depression, and suicidal propensity.

The study was conducted in compliance with the Declaration of Helsinki and its amendments,${ }^{34}$ and the norms of the local institutional review board. The experimental protocol $(0588 / 07)$ was approved by the local research ethics committee and all patients provided their written informed consent. This trial is registered at http://www.ClinicalTrials. gov/ (NCT01452321).

The participants were randomized to one of two groups, ie, for active rTMS (eight participants, mean age $29.63 \pm 6.37$ years) or for sham stimulation (six participants, mean age $26.67 \pm 7.15$ years). Unrestricted randomization was performed using a computerized random-number generator (random.org) according to CONSORT guidelines. Subjects assigned to the active rTMS group received repetitive stimulation at $120 \%$ of the motor threshold for 4 weeks (5 days/week). Each weekday, the subjects received $5 \mathrm{~Hz}$ stimulations as follows: 25 trains (1250 pulses) per day, each train lasting 10 seconds, with 20 -second intervals between trains. ${ }^{35}$ Stimulation was generated over the left dorsolateral prefrontal cortex, which was assumed to be exactly $5 \mathrm{~cm}$ rostral to the point of optimal stimulation for the right abductor pollicis brevis muscle in the left parasagittal plane. ${ }^{36} \mathrm{We}$ used an eight-shape coil perpendicular to an imaginary line extending from the point of stimulation to the nose of the participant; sham stimulation followed the same protocol, but using a placebo coil. The stimulation was performed using a high-speed magnetic stimulator (MagVenture; Medtronic, Minneapolis, MN). The motor threshold of the right abductor pollicis brevis (a thumb muscle) was determined, as described elsewhere. ${ }^{36}$ Patients in the placebo group were provided with the option to receive active treatment immediately after completion of the blind phase.

\section{Evaluation of clinical, neurocognitive, and social performance}

A board-certified neuropsychologist, who was blinded to the findings of the other raters and to the study group assignment (did not had access to the laboratory where rTMS treatment was administered or to records containing patient data and study procedures), conducted the clinical, neurocognitive, and social performance evaluation on the day before initiation of treatment (baseline), after 4 weeks 
(week 4, end of treatment), and at follow-up (week 6, 2 weeks after treatment). The two main outcome measures were changes in HDRS $/ 17^{32}$ and $\operatorname{EPDS}^{33}$ scores. A positive response to treatment was defined as a reduction of at least $30 \%$ in HDRS/17 and EPDS scores.

\section{Clinical evaluation}

The clinical evaluation included the HDRS/17 and the EPDS; the 14 item-Hamilton Anxiety Scale (HARS/14) ${ }^{37}$; the Clinical Global Impression (CGI, 1-Global Impression of Severity, 2-Global Impression of Change and 3-Quality of Life $^{37}$ ); the Global Assessment Scale ${ }^{37}$; and the Medical Outcomes Study 36-item Short-Form Health Survey (SF-36)Quality of Life (in order to quantify health-related quality of life, comprising eight fields: functional capacity, physical aspects, pain, general health, vitality, social aspects, emotional aspects, and mental health ${ }^{38}$ ).

\section{Neurocognitive assessment}

The neurocognitive battery was comprised of the following tests: Executive function - Trail Making Test (parts A and B); Wisconsin Card Sorting Test (completed number of categories, total errors, and perseverative errors); Controlled Oral Word Association Test (phonemic category: letters F/A/S and semantic category: animals); Victoria Stroop Test (colors, color-word, and color-word interference). Learning through immediate memory ability - Rey Auditory Verbal Learning Test (immediate recall: better performance in trials list A and total performance of 5-trials list A; retention: trial A6 and delayed recall: trial A7). ${ }^{39,40}$ Other specific cognitive functions - WAIS-III [adapted for use in Brazil] - subtests: similarities (abstract verbal reasoning), picture completion (ability to perceive visual details quickly), digit span forward, backward and total (attention, concentration, mental control, working memory) and coding (visual-motor coordination, motor and mental speed, visual working memory). ${ }^{41}$

This neurocognitive battery was defined based on two perspectives: the importance of each of these tests to the neuropsychological evaluation of subjects suffering with affective disorders, especially depression, ${ }^{26}$ and the skills that are critical to motherhood, particularly in light of the necessity to cope with the stress that stems from the psychological conditions associated with the postpartum period..$^{42}$

\section{Social performance}

In order to evaluate changes in social performance, we used the Social Adjustment Scale-Self Report, adapted for use in Brazil. ${ }^{43}$ This scale comprises six independent behavioral subscales (work at home [house activities], social/leisure [social life and leisure activities], extended family-primary relationship [relationships with family members], parental [relationship with spouse or partner], unit-child [relationship with children], and financial situation [current economic condition], which generate an index of overall social performance). The Social Adjustment Scale-Self Report is a self-report scale that was administered while a certified evaluator was observing but not interacting with the subjects.

\section{Statistical analysis}

Demographic and diagnostic data and baseline scores on rating scales were compared between groups using the Student's $t$-test, non-parametric Wilcoxon-Mann-Whitney test, or Fisher's exact test, as applicable. Fisher's exact test was used to compare the two groups in terms of the proportion of patients in each group. For all other outcome measures, as well as for the neuropsychological battery test scores, the effects of group, time, and group $\times$ time interaction (longitudinal comparison between the groups) were determined using repeated-measures analysis of variance (ANOVA). In order to evaluate treatment efficacy, mean and the standard deviation for each variable are reported in three different moments (baseline, week 4, and week 6). Interaction effects tests are also reported (baseline versus week 4, week 4 versus week 6 , and baseline versus week 6). The residual normality assumption of the model was confirmed by the Kolmogorov-Smirnov test. We performed Bonferroni correction, as well as a post hoc correction for multiple testing, using the false discovery rate, which is generally considered to be most appropriate in this context. ${ }^{44}$

All tests were two-tailed, and the level of significance was set at $P<0.05$. Statistical analysis was performed using the Statistical Package for Social Sciences version 19.0 for Windows (SPSS IBM Corporation, Armonk, NY). Only patients who completed the study were included in the analysis.

\section{Results}

\section{Demographic and clinical profile}

Of the 14 patients included in the study, all completed the study (all breastfed their babies at least until week 6) and were included in analysis. At baseline, both groups were comparable in terms of demographic and psychopathological variables (active rTMS, $\mathrm{n}=8$; sham rTMS, $\mathrm{n}=6$ ).

The ABIPEME (Association of Marketing Research Firms in Brazil) classification system subdivides the Brazilian population into categories according to socioeconomic status. It includes items addressing material possessions and 
educational level of the family members. The socioeconomic classification is divided in five classes, ie, A, B, C, D, and E, in accordance with the scores achieved for material possessions and educational level.

In relation to diagnosis and history of disease, three patients in the active rTMS group and two patients in the sham rTMS group were experiencing their first depressive episode; two patients in the active rTMS group and two patients in sham rTMS group suffered from recurrent depression (with several prior episodes); and three patients in active rTMS group and two patients in sham rTMS group were experiencing their first bipolar depressive episode. The demographic and clinical profiles of the two groups are shown in Table 1.

\section{Clinical, neurocognitive, and social performance}

Assessment of the effectiveness of blinding did not show results significantly different from what would be expected by chance. The rater guessed the assignment correctly in 54\% of cases, whereas $60 \%$ of the patients guessed correctly when asked about which group they believed they had been assigned to. This result confirmed that the blinding procedure was efficient.

Table 2 shows the mean differences in scores between the groups at different weeks, regarding the measures of clinical, neuropsychological, and social performance. The active rTMS group but not the control group showed a positive response to treatment, with a reduction of at least $30 \%$ in HDRS/17 and EPDS scores at week 6 (after 2 weeks of follow-up).

\section{Clinical psychiatric scales}

In comparison with the sham group, the active rTMS group showed statistically significant differences in scores at baseline versus week 6 for the HDRS/17 $(P=0.020)$, Global Assessment Scale $(P=0.037)$, and CGI1 $(P=0.047)$, when using the false discovery rate.

Although the active group showed a reduction of $39.4 \%$ in EPDS against 6.2\% in the placebo group, this difference did not reach statistical significance. With respect to the HARS/14 (28.1\% versus 6.6\%), CGI3 (44.2\% versus 26.3\%), SF-36 for vitality ( $80.0 \%$ versus $17.9 \%$ ) and mental health (102.3\% versus $8.6 \%$ ), no difference was observed for any interaction effects comparing the active and sham treatments when using the false discovery rate (Table 2), but statistically significant differences were observed using analysis of variance without false discovery rate correction; the values were HDRS $/ 17(P=0.001)$, EDPS $(P=0.007), \mathrm{HARS} / 14$ $(P=0.012)$, Global Assessment Scale $(P=0.002$; also in baseline versus week $4, P=0.025)$, CGI1 $(P=0.004)$ and CGI3 $(P=0.038)$, and SF-36 for vitality $(P=0.054)$, and mental health $(P=0.054$; Table 2 and Figure 1$)$.

\section{Neuropsychological battery}

Between-group comparison associated with application of false discovery rate correction did not reveal statistically significant differences in performance for the Trail Making Test-B (31.4\% versus $12.9 \%)$ or in the Victoria Stroop TestInterference $(31.7 \%$ versus $10.0 \%)$. When using analysis of variance without false discovery rate correction, statistical differences appeared in both tests, ie, Trail Making Test-B (baseline versus week $4, P=0.039$ ) and Victoria Stroop Test-Interference (baseline versus week 6, $P=0.034$, Table 2 and Figure 1). No statistically significant differences were observed for the rest of the neuropsychological evaluation.

\section{Social performance}

Regarding the Social Adjustment Scale Self-Report, the groups differed at baseline in overall social performance (active rTMS $128.13 \pm 14.09$ versus sham rTMS $106.00 \pm 15.77$ $P=0.017)$. This scenario was reversed over the course of treatment. At week 6 (follow-up), the active rTMS group had improved general social performance scores by $25.5 \%$ $(95.50 \pm 32.00)$, whereas the sham group (102.00 \pm 33.28$)$ had a $3.8 \%$ improvement. This trend was not considered significant under false discovery rate control, although it was significant under the analysis of variance $(P=0.050)$. Additionally, in the Social Adjustment Scale Self-ReportWork-at-Home, a statistically significant improvement was observed in both false discovery rate and analysis of variance analysis (baseline versus week $6, P=0.020$ and $P=0.001$, respectively; and also in baseline versus week $4, P=0.035$; Table 2 and Figure 1).

\section{Side effects}

Subjects reported no significant side effects during the study. Two patients complained of minor scalp discomfort during application and/or mild headache immediately after their rTMS sessions.

\section{Discussion}

\section{Extent to which rTMS enhances clinical, social, and cognitive functioning}

In this study, stimulatory magnetic pulses $(5 \mathrm{~Hz})$ were delivered to the left dorsolateral prefrontal cortex in the active treatment group. Despite the fact that this area of the brain is involved in affective, social, and cognitive processes (due 
Table I Demographic data, diagnostic/clinical data, and baseline rating scale scores for patients receiving repetitive transcranial magnetic stimulation (active or sham group)*

\begin{tabular}{|c|c|c|c|}
\hline Characteristic & $\begin{array}{l}\text { Active rTMS } \\
n=8\end{array}$ & $\begin{array}{l}\text { Sham rTMS } \\
n=6\end{array}$ & $P$-value \\
\hline Age of the mother (years) & $29.63 \pm 6.37$ & $26.67 \pm 7.15$ & $0.440^{\mathrm{a}}$ \\
\hline Age of the infant (months) & $4.13 \pm 2.85$ & $3.50 \pm 2.74$ & $0.507^{b}$ \\
\hline Maternal years of schooling & $12.75 \pm 2.55$ & $11.50 \pm 2.74$ & $0.403^{\mathrm{a}}$ \\
\hline Number of infant & $1.50 \pm 0.76$ & $1.00 \pm 0.00$ & $0.105^{b}$ \\
\hline \multicolumn{4}{|l|}{ Socioeconomic class (ABIPEME) } \\
\hline $\mathrm{A}$ or $\mathrm{B}, \mathrm{n}(\%)$ & $3(37.5)$ & $2(33.3)$ & $0.334^{c}$ \\
\hline C, n (\%) & $4(50)$ & I (I6.7) & \\
\hline $\mathrm{D}$ or $\mathrm{E}, \mathrm{n}(\%)$ & I (I2.5) & $3(50)$ & \\
\hline \multicolumn{4}{|l|}{ Marital status** } \\
\hline Partner, n (\%) & $8(100)$ & $2(33.3)$ & $0.015^{c}$ \\
\hline No partner, n (\%) & $0(0)$ & $4(66.7)$ & \\
\hline \multicolumn{4}{|l|}{ Primiparity } \\
\hline First infant, n (\%) & $5(62.5)$ & $6(100)$ & $0.472^{c}$ \\
\hline Second infant, n (\%) & $2(25.0)$ & $0(0)$ & \\
\hline Third infant, n (\%) & $\mathrm{I}(12.5)$ & $0(0)$ & \\
\hline \multicolumn{4}{|l|}{ DSM-IV diagnosis (via the structured clinical interview) } \\
\hline Major depressive disorder - onset in postpartum, $\mathrm{n}(\%)$ & $3(37.5)$ & $2(33.3)$ & $1,000^{c}$ \\
\hline Major depressive disorder - recurrent episode, n (\%) & $2(25)$ & $2(33.3)$ & \\
\hline Affective bipolar disorder II - depressive episode in postpartum, n (\%) & $3(37.5)$ & $2(33.3)$ & \\
\hline \multicolumn{4}{|l|}{ Onset of depressive symptoms } \\
\hline Up to 5 days postpartum, $\mathrm{n}(\%)$ & $8(100)$ & $4(66.7)$ & $0.164^{c}$ \\
\hline After I month postpartum, n (\%) & $0(0)$ & I (I6.7) & \\
\hline Between the 2nd and the 6th month after delivery, $\mathrm{n}(\%)$ & $0(0)$ & I (I6.7) & \\
\hline \multicolumn{4}{|l|}{ Previous depressive episodes } \\
\hline Yes, n (\%) & $5(62.5)$ & $3(50)$ & $1.000^{c}$ \\
\hline No, n (\%) & $3(37.5)$ & $3(50)$ & \\
\hline \multicolumn{4}{|l|}{ Previous hypomania episodes } \\
\hline Yes, n (\%) & $\mathrm{I}(\mathrm{I} 2.5)$ & $2(33.3)$ & $0.538^{c}$ \\
\hline No, n (\%) & $7(87.5)$ & $4(66.7)$ & \\
\hline \multicolumn{4}{|l|}{ Previous psychopharmacological treatment } \\
\hline Yes, n (\%) & $4(50)$ & $0(0)$ & $0.084^{c}$ \\
\hline No, n (\%) & $4(50)$ & $6(100)$ & \\
\hline \multicolumn{4}{|l|}{ Family history } \\
\hline Yes, n (\%) & $6(75)$ & $4(66.7)$ & $1.000^{c}$ \\
\hline No, n (\%) & $2(25)$ & $2(33.3)$ & \\
\hline \multicolumn{4}{|l|}{ Medication in use: clonazepam ( $\mathrm{l}$ mg/day), $\mathrm{n}(\%)$} \\
\hline Yes, n (\%) & $3(37.5)$ & $0(0)$ & $0.209^{c}$ \\
\hline No, n (\%) & $5(62.5)$ & $6(100)$ & \\
\hline \multicolumn{4}{|l|}{ Baseline rating scale scores } \\
\hline HDRS/I7 score & $29.13 \pm 5.64$ & $26.67 \pm 5.68$ & $0.437^{a}$ \\
\hline EPDS score & $22.50 \pm 3.16$ & $21.50 \pm 3.73$ & $0.597^{\mathrm{a}}$ \\
\hline HARS/I4 score & $35.13 \pm 8.39$ & $32.83 \pm 7.39$ & $0.605^{\mathrm{a}}$ \\
\hline CGII score & $4.88 \pm 0.35$ & $4.67 \pm 0.52$ & $0.365^{b}$ \\
\hline GAS score & $38.88 \pm 7.70$ & $43.00 \pm 7.29$ & $0.33 \mathrm{I}^{\mathrm{a}}$ \\
\hline SF-36-vitality score & $28.13 \pm 17.72$ & $23.33 \pm 16.02$ & $0.605^{a}$ \\
\hline SF36-mental health score & $22.00 \pm 13.86$ & $23.33 \pm 20.15$ & $0.885^{a}$ \\
\hline
\end{tabular}

Notes: ${ }^{a}$ Student test; ${ }^{b}$ Wilcoxon-Mann-Whitney test; 'Fisher's exact test; *data presented in terms of mean \pm standard deviation (SD), unless otherwise specified; $* *$ statistically significant difference, Fisher's exact test.

Abbreviations: rTMS, repetitive Transcranial Magnetic Stimulation; ABIPEME, Associação Brasileria de Institutos de Pesquisa de Mercado (Association of Marketing Research Firms in Brazil); DSM-IV, Diagnostic and Statistical Manual of Mental Disorders, Fourth Edition; HDRS/I7, I7-item Hamilton Depression Rating Scale; EPDS, Edinburgh Postnatal Depression Scale; HARS/ I4, I4-item Hamilton Anxiety Rating Scale; CGI, Clinical Global Impression - CGII-Global Impression of Severity; GAS, Global Assessment Scale; SF-36, Medical Outcomes Study 36-item Short-form Health Survey. 


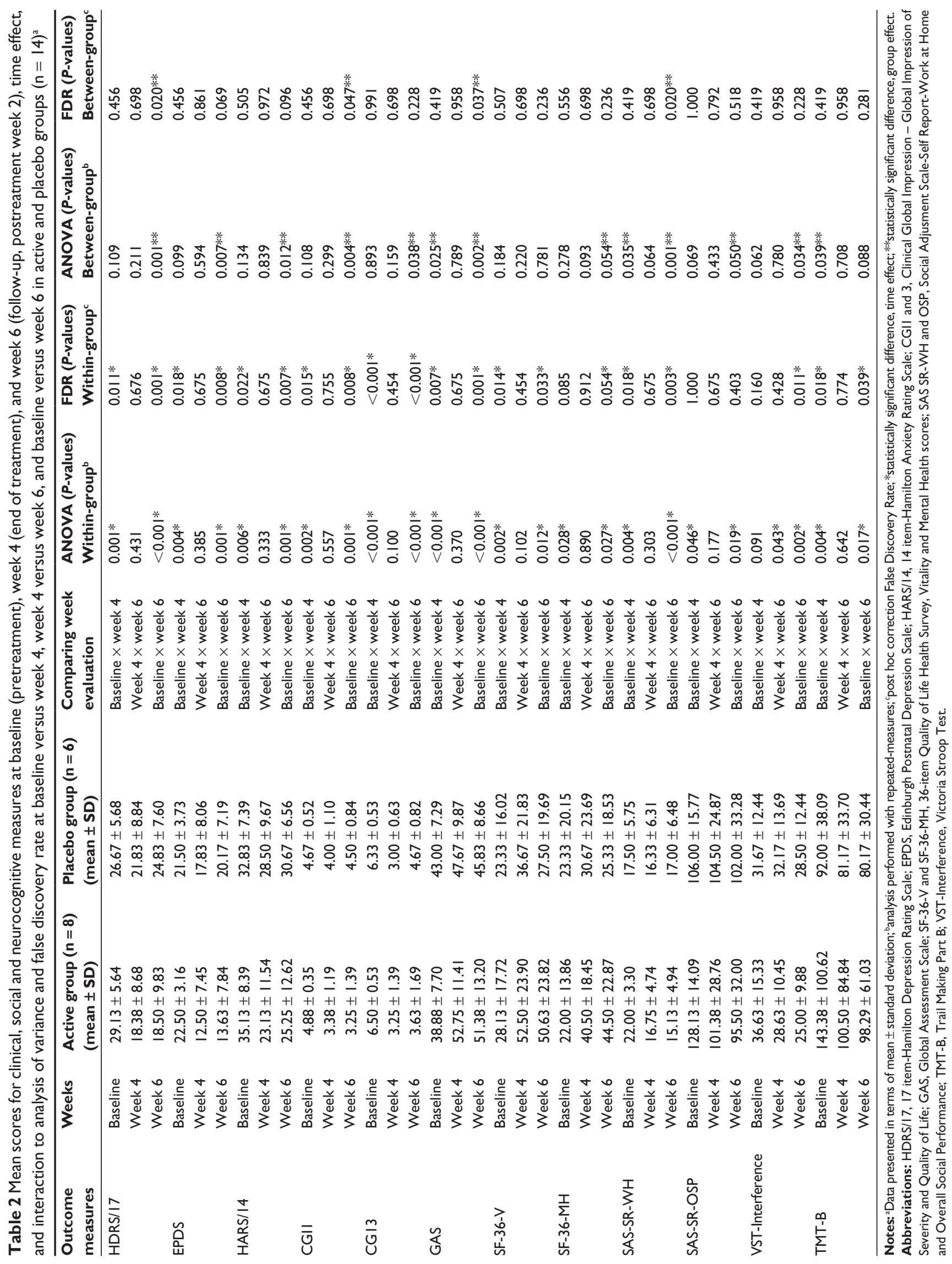



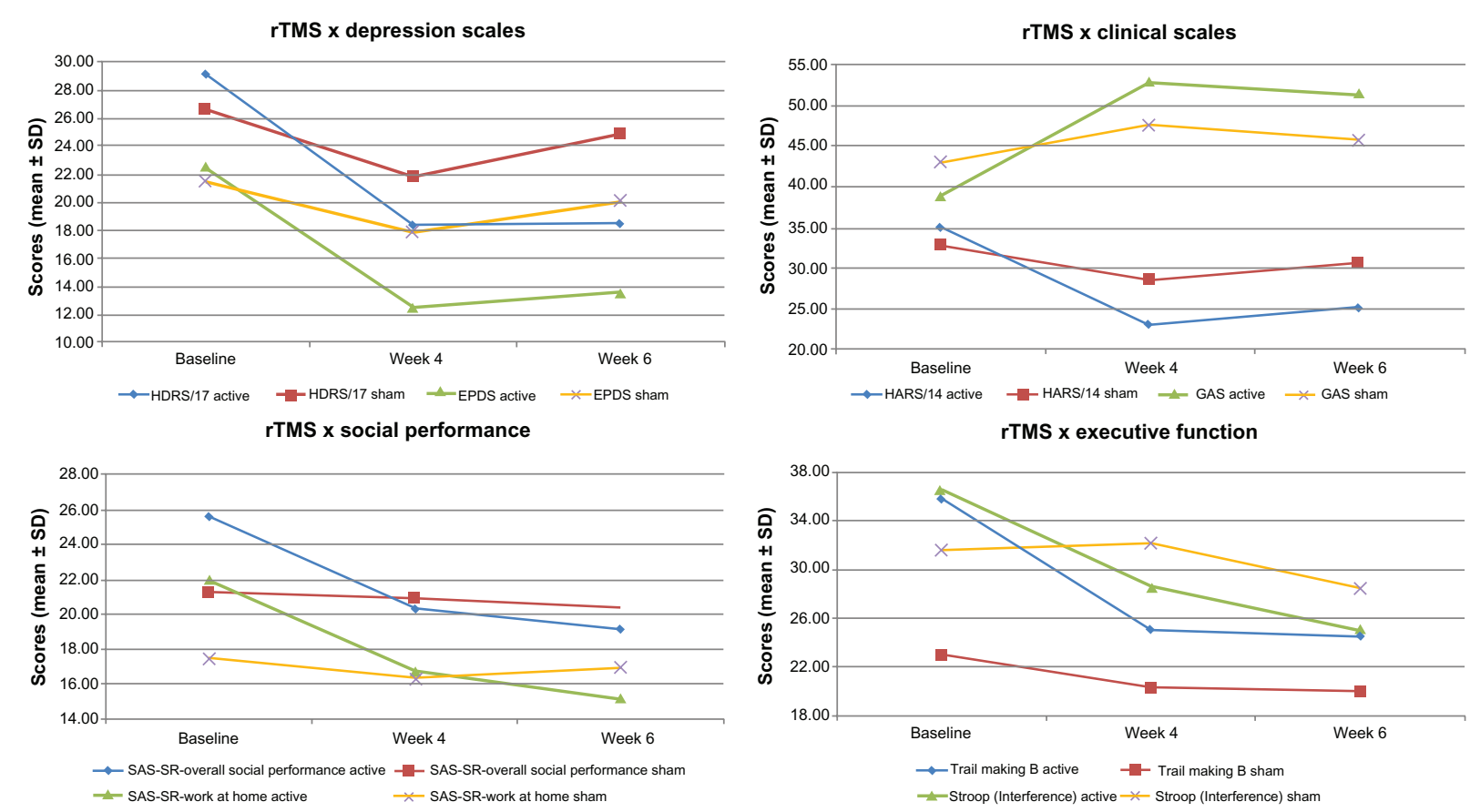

Figure I Change in mean values of clinical scales and neuropsychological tests at baseline (pre-treatment), weeks 4 (end of treatment) and 6 (follow up: post-treatment week 2) for active and placebo groups.

Abbreviations: rTMS, repetitive Transcranial Magnetic Stimulation; HDRS/17, 17-item-Hamilton Depression Rating Scale; EPDS, Edinburgh Postnatal Depression Scale; HARS/I4, 14 item-Hamilton Anxiety Rating Scale; GAS, Global Assessment Scale; SAS-SR, Social Adjustment Scale-Self Report-Overall Social Performance and Work at Home; Trail Making B and Stroop Test (Interference).

to its highly connected structure), only marginal gains were observed in relation to social and cognitive dimensions.

There are several possible explanations for these findings, ie, that clinical dimensions tend to be more sensitive than cognitive and social performance to the effects of rTMS due to the spread of activation in frontolimbic networks, and that the clinical effects were stronger than the other two in this particular case because this dimension was the most affected at baseline. Adding to that, one could speculate that some results in this study became nonsignificant when switching from analysis of variance to Bonferroni testing, because this method increases the type II (false negative) error rate. ${ }^{44}$ When we applied the false discovery rate, some significant results reappeared.

\section{Skills essential for motherhood}

rTMS did not produce any significant improvements in cognitive performance, although there were some nonsignificant results pointing in this direction. It is worth noting that the observed moderate cognitive enhancement does not appear to reflect rehearsal, because both groups had the same opportunity to learn the tasks (this being a placebo-controlled study), whereas improvement was only observed in the active group. Considering that abilities such as memory, motor skills, mental flexibility, attention, and abstract learning are essential for parenting, ${ }^{45}$ we hypothesize that improvements in performance can enhance the capacity to cope with the challenges of the puerperium and exert a protective effect against pathological levels of stress for the mother and child, therefore inhibiting the chronification of depression. ${ }^{46}$

\section{Cumulative effect of rTMS}

We observed marginal cumulative effects of rTMS on affective, social, and cognitive dimensions from week 4 to week 6 . In this regard, the most promising results were found in relation to performance on the HDRS/17 and Social Adjustment Scale Self-Report-Work-at-Home $(P=0.020$ for both). This finding is interesting when considering that PPD is defined in light of very strong social bonds (reported by the family), which are not only dependent on the ability of the mother to be supportive in the affective domain, but also on her capacity to cope with practical issues related to family reorganization.

\section{Limitations of this study}

The main limitations of this study are that the sample size is not large and the marital status of the mothers was not standardized, with the inclusion of four women who had 
no partner during the time this study was performed. These single mothers were randomized to the sham rTMS group. It is important to note that absence of a partner represents a risk factor for PPD, so that depression could be more remarkable in this group. ${ }^{5}$ However, this did not occur, as shown by equivalence in baseline performance on the HDRS/17 and EPDS between the groups. Another potential limitation relates to the fact that some patients suffered from recurrent depressive disorder. Finally, it was not possible to determine the extent to which the marginal cognitive and social improvements that were found represent byproducts of the clinical efficacy of the method.

\section{Conclusion}

This paper presents the results of the first double-blind, controlled pilot trial on the effects of $5 \mathrm{~Hz} \mathrm{rTMS}^{35}$ on clinical, social, and cognitive functioning in patients with PPD. At the same time as confirming improvement in affective and specific social domains, the study also provides preliminary evidence that rTMS produces small gains in overall social and specific cognitive performance. These social improvements may be strategic to the family in several ways, given that the puerperium involves decisive social demands associated with changes in family and household organization. Being unfit to cope with the practical demands of the puerperium can have deleterious effects that go beyond the initial period, affecting the child and perpetuating depression throughout generations, by both epigenetic and behaviorally mediated mechanisms. ${ }^{47,48}$

This study suggests that a paradigm shift in the treatment of PPD may be both desirable and feasible. rTMS produces results that are comparable with those of antidepressants, without the necessity to cease breastfeeding or transmit undesirable chemical compounds to the newborn. If corroborated by clinical trials involving larger numbers of patients, this type of study may emerge as pioneering rTMS as a treatment of choice for PPD.

\section{Disclosure}

The authors report no conflicts of interest in this work.

\section{References}

1. O'Hara MW. Postpartum depression: what we know. J Clin Psychol. 2009; 65(12): 1258-1269.

2. Angst J, Gamma A, Rossler W, Ajdacic V, Klein DN. Long-term depression versus episodic major depression: results from the prospective Zurich study of a community sample. J Affect Disord. 2009;115(1-2):112-121.

3. Halbreich U, Karkun S. Cross-cultural and social diversity of prevalence of postpartum depression and depressive symptoms. J Affect Disord. 2006;91(2-3):97-111.
4. Caspi A, Sugden K, Poulton R, et al. Influence of life stress on depression: moderation by a polymorphism in the 5-HTT gene. Science. 2003;301(5631):386-389.

5. Christie AL, Katherine JG, HeatherAF, HarimY, Sheila MM, Matthew MD. Risk factors for depressive symptoms during pregnancy: a systematic review. Am J Obstet Gynecol. 2010;202(1):5-14.

6. Ryan D, Milis L, Misri N. Depression during pregnancy. Can Fam Physician. 2005;51:1087-1093.

7. Goodman SH. Depression in mothers. Annu Rev Clin Psychol. 2007;3: $107-135$.

8. Hammen C, Shih J, Altman T, Brennan PA. Interpersonal impairment and the prediction of depressive symptoms in adolescent children of depressed and nondepressed mothers. J Am Acad Child Adolesc Psychiatry. 2003;42(5):571-577.

9. Hammen C, Brennan PA. Severity, chronicity, and timing of maternal depression and risk for adolescent offspring diagnoses in a community sample. Arch Gen Psychiatry. 2003;60(3):253-258.

10. Goodman SH, Gotlib IH. Risk for psychopathology in the children of depressed mothers: a developmental model for understanding mechanisms of transmission. Psychol Rev. 1999;106(3):458-490.

11. Choi Y, Bishai D, Minkovitz CS. Multiple births are a risk factor for postpartum maternal depressive symptoms. Pediatrics. 2009;123(4):1147-1154.

12. Toohey J. Depression during pregnancy and postpartum. Clin Obstet Gynecol. 2012;55(3):788-797.

13. Turner EH, Matthews AM, Linardatos E, Tell RA, Rosenthal R. Selective publication of antidepressant trials and its influence on apparent efficacy. $N$ Engl J Med. 2008;358(3):252-260.

14. Payne JL. Antidepressant use in the postpartum period: practical considerations. Am J Psychiatry. 2007;164(9):1329-1332.

15. Kirsch I, Deacon BJ, Huedo-Medina TB, Scoboria A, Moore TJ, Johnson BT. Initial severity and antidepressant benefits: a meta-analysis of data submitted to the Food and Drug Administration. PLoS Med. 2008;5(2):e45.

16. Campagne DM. Fact: antidepressants and anxiolytics are not safe during pregnancy. European J Obstet Gynecol Reprod Biol. 2007; 135(2):145-148.

17. Moscrip TD, Terrace HS, Sackeim HA, Lisanby SH. Randomized controlled trial of the cognitive side-effects of magnetic seizure therapy (MST) and electroconvulsive shock (ECS). Int J Neuropsychopharmacol. 2006; 9(1):1-11.

18. Fitzgerald P. Repetitive transcranial magnetic stimulation and electroconvulsive therapy: complementary or competitive therapeutic options in depression? Australas Psychiatry. 2004;12(3):234-238.

19. Loo CK, McFarquhar TF, Mitchell PB. A review of the safety of repetitive transcranial magnetic stimulation as a clinical treatment for depression. Int J Neuropsychopharmacol. 2008;11(1):131-147.

20. Hallett M. Transcranial magnetic stimulation and the human brain. Nature. 2000;406(6792):147-150.

21. Nahas Z, Teneback CC, George MS, et al. Brain effects of TMS delivered over prefrontal cortex in depressed adults: role of stimulation frequency and coil-cortex distance. $J$ Neuropsychiatry Clin Neurosci. 2001;13(4):459-470.

22. Kudo K, Yamada M, Takahashi K, et al. Repetitive transcranial magnetic stimulation induces $\mathrm{kf}-1$ expression in the rat brain. Life Sci. 2005;76(21):2421-2429.

23. Holtzheimer PE, Russo J, Avery DH. A meta-analysis of repetitive transcranial magnetic stimulation in the treatment of depression. Psychopharmacol Bull. 2001;35(4):149-169.

24. Schutter DJ. Quantitative review of the efficacy of slow-frequency magnetic brain stimulation in major depressive disorder. Psychol Med. 2010;40(11):1789-1795.

25. Garcia KS, Flynn P, Pierce KJ, Claudle M. Repetitive transcranial magnetic stimulation treats postpartum depression. Brain Stimul. 2010;3(1):36-41.

26. Guse B, Falkai P, Wobrock T. Cognitive effects of high-frequency repetitive transcranial magnetic stimulation: a systematic review. J Neural Transm. 2010;117(1):105-122. 
27. Barr MS, Farzan F, Rusjan PM, Chen R, Fitzgerald PB, Daskalakis ZJ. Potentiation of gamma oscillatory activity through repetitive transcranial magnetic stimulation of the dorsolateral prefrontal cortex. Neuropsychopharmacology. 2009;34(11):2359-2367.

28. Preston G, Anderson E, Silva C, Goldberg T, Wassermann E. Effects of $10 \mathrm{~Hz}$ rTMS on the neural efficiency of working memory. $J \operatorname{Cogn}$ Neurosci. 2010;22(3):7-56.

29. Allen NB, Badcock PBT. Darwinian models of depression: a review of evolutionary accounts of mood and mood disorders. Prog Neuropsychopharmacol Biol Psychiatry. 2006;30(5):815-826.

30. Hagen EH. The functions of postpartum depression. Evol Hum Behav. 1999;20(5):325-359.

31. First MB, Spitzer RL, Gibbon M, Williams JBW. Structured Clinical Interview for DSM-IV Axis I Disorders, Patient Edition (SCID-P, Version 2.0). New York, NY: New York State Psychiatric Institute, Biometrical Research; 1996.

32. Blacker D. Psychiatric rating scales. In: Sadock B, Sadock V, editors. Comprehensive Textbook of Psychiatry. 7th ed. Philadelphia, PA: Lippincott Williams \& Wilkins; 2000.

33. Cox JL. Postnatal Depression. A Guide for Health Professionals. Edinburgh, UK: Churchill Livingstone; 1986.

34. Lewis J, Jonsson B, Kreutz G, Sampaio C, Van Zwieten-Boot B. Placebo-controlled trials and the Declaration of Helsinki. Lancet. 2002;359(9314):1337-1340.

35. Rumi DO, Gattaz WF, Marcolin MA, et al. Transcranial magnetic stimulation accelerates the antidepressant effect of amitriptyline in severe depression: a double-blind placebo-controlled study. Biol Psychiatry. 2005;57(2):162-166.

36. Pascual-Leone A, Rubio B, Pallardó F, Catalá M. Rapid-rate transcranial magnetic stimulation of left dorsolateral prefrontal cortex in drug-resistant depression. Lancet. 1996;348(9022):233-237.

37. Mansur CG, Myczkowki ML, Marcolin MA, et al. Placebo effect after prefrontal magnetic stimulation in the treatment of resistant obsessivecompulsive disorder: a randomized controlled trial. Int J Neuropsychopharmacol. 2011;14(10):1389-1397.
38. Campolina AG, Ciconelli RM.HYPERLINK "http://www.ncbi. nlm.nih.gov/pubmed/18604180" SF-36 and the development of new assessment tools for quality of life. Acta Reumatol Port. 2008 Apr-Jun;33(2):127-33. Review. Portuguese.

39. Lezak MD. Neuropsychological Assessment. 3rd ed. New York, NY: Oxford University Press; 1995.

40. Spreen O, Strauss E. A Compendium of Neuropsychological Tests. New York, NY: Oxford University Press; 1998.

41. Wechsler D. Manual for the Wechsler Adult Intelligence Scale-Revised, Brazilian Adaptation. Sao Paulo, Brazil: Casa do Psicologo; 2004.

42. Ngai FW, Chan SW. Psychosocial factors and maternal wellbeing: an exploratory path analysis. Int J Nurs Stud. 2011;48(6):725-731.

43. Gorenstein C, Moreno RA, Andrade L, et al. Validation of the Portuguese version of the Social Adjustment Scale on Brazilian samples. J Affect Disord. 2002;69(1-3):167-175.

44. Benjamini Y, Hochberg Y. Controlling the false discovery rate: a practical and powerful approach to multiple testing. J.R. Statisti. Soc. B. 1995;57:289-300.

45. Workman JL, Barha CK, Galea LA. Endocrine substrates of cognitive and affective changes during pregnancy and postpartum. Behav Neurosci. 2012;126(1):54-72.

46. Kingston D, Tough S, Whitfield H. Prenatal and postpartum maternal psychological distress and infant development: a systematic review. Child Psychiatry Hum Dev. 2012;43(5):683-714.

47. Letourneau NL, Dennis CL, Watson W, et al. Postpartum depression is a family affair: addressing the impact on mothers, fathers, and children. Issues Ment Health Nurs. 2012;33(7):445-457.

48. Conroy S, Pariante CM, Moran P, et al. Maternal psychopathology and infant development at 18 months: the impact of maternal personality disorder and depression. J Am Acad Child Adolesc Psychiatry. 2012;51(1):51-61.
Neuropsychiatric Disease and Treatment

\section{Publish your work in this journal}

Neuropsychiatric Disease and Treatment is an international, peerreviewed journal of clinical therapeutics and pharmacology focusing on concise rapid reporting of clinical or pre-clinical studies on a range of neuropsychiatric and neurological disorders. This journal is indexed on PubMed Central, the 'PsycINFO' database and CAS.

\section{Dovepress}

The manuscript management system is completely online and includes a very quick and fair peer-review system, which is all easy to use. Visit http://www.dovepress.com/testimonials.php to read real quotes from published authors. 